\title{
Correction to: Plant Phosphopeptide Identification and Label-Free Quantification by MaxQuant and Proteome Discoverer Software
}

\author{
Shalan Li, Haitao Zan, Zhe Zhu, Dandan Lu, and Leonard Krall
}

Correction to:

Chapter 13 in: Xu Na Wu (ed.), Plant Phosphoproteomics:

Methods and Protocols, Methods in Molecular Biology, vol. 2358, https://doi.org/10.1007/978-1-0716-1625-3_13

In the original version of this book, the title of chapter 13 was published with a typographical error. It has now been rectified in the revised version of this book. 